PHYSICAL REVIEW B 85, 014433 (2012)

\title{
Stochastic form of the Landau-Lifshitz-Bloch equation
}

\author{
R. F. L. Evans, ${ }^{1}$ D. Hinzke, ${ }^{2}$ U. Atxitia, ${ }^{3}$ U. Nowak,${ }^{2}$ R. W. Chantrell, ${ }^{1}$ and O. Chubykalo-Fesenko ${ }^{3}$ \\ ${ }^{1}$ Department of Physics, University of York, Heslington, York YO10 5DD, United Kingdom \\ ${ }^{2}$ Universität Konstanz, Fachbereich Physik, Universitatssträße 10, D-78464 Konstanz, Germany \\ ${ }^{3}$ Instituto de Ciencia de Materiales de Madrid, CSIC, Cantoblanco, E-28049 Madrid, Spain
}

(Received 20 October 2011; published 26 January 2012)

\begin{abstract}
The Landau-Lifshitz-Bloch equation is a formulation of dynamic micromagnetics valid at all temperatures, treating both the transverse and longitudinal relaxation components important for high-temperature applications. In this paper we discuss two stochastic forms of the Landau-Lifshitz-Bloch equation. Both of them are consistent with the fluctuation-dissipation theorem. We derive the corresponding Fokker-Planck equations and show that only the stochastic form of the Landau-Lifshitz-Bloch equation proposed in the present paper is consistent with the Boltzmann distribution at high temperatures. The previously used form does not satisfy this requirement in the vicinity of the Curie temperature. We discuss the stochastic properties of both equations and present numerical simulations for distribution functions and the average magnetization value as a function of temperature.
\end{abstract}

DOI: 10.1103/PhysRevB.85.014433

\section{INTRODUCTION}

Stochastic fluctuations were introduced into magnetism by Brown,,${ }^{1,2}$ who recognized the necessity to describe the deviations from the average magnetization trajectory in an ensemble of noninteracting nanoparticles described as macrospins within the Landau-Lifshitz-Gilbert (LLG) equation of motion. $\mathrm{He}$ introduced fluctuating fields, formal quantities responsible for magnetization fluctuations. The strengths of these fields were calculated based on two approaches with the same final results: (i) the fluctuation-dissipation theorem (FDT); (ii) deriving the corresponding Fokker-Planck (FP) equation for the probability distribution function and requiring that its equilibrium solution be the Boltzmann distribution. Brown's idea has important consequences in the theory of magnetic nanoparticles, since it allows the calculation of the switching probability over energy barriers created by magnetic anisotropy and therefore to describe the effect of superparamagnetism., ${ }^{2,3}$ Later the results of Brown were brought to micromagnetic modeling, ${ }^{4}$ understanding each discretization unit as a macrospin with fluctuating magnetization direction and giving rise to thermal micromagnetics. ${ }^{4,5}$

One should note that the LLG equation imposes a serious restriction in the magnetization dynamics since it assumes a constant magnetization length. ${ }^{6}$ It has been shown that the stochastic micromagnetic approach based on the LLG equation significantly overestimates the Curie temperature ${ }^{7}$ because the high-frequency spin waves are artificially truncated. Based on the thermal averaging of many exchange-coupled atomistic spins, Garanin ${ }^{8}$ derived the Landau-Lifshitz-Bloch (LLB) equation. The LLB equation essentially interpolates between the LLG equation at low temperatures and the GinzburgLandau theory of phase transitions. It is valid not only below but also above the Curie temperature $T_{\mathrm{c}}$. An important property of the LLB equation is that the magnetization magnitude is no longer conserved but is a dynamical variable. The transverse and the longitudinal magnetization components have different damping parameters which can be related to the microscopic damping ("coupling to the bath") parameter. Thus the thermal fluctuations on average are included in the temperature dependence of macroscopic parameters such as magnetization, anisotropy, and damping.
PACS number(s): 75.40.Mg, 75.78.Cd, 75.60.Jk

LLB micromagnetics has become a real alternative to LLG micromagnetics for temperatures which are close to the Curie temperature $\left(T \gtrsim 3 T_{\mathrm{c}} / 4\right) .{ }^{9}$ This is realistic for some novel exciting phenomena, such as light-induced demagnetization with powerful fs lasers. ${ }^{10-12}$ During this process the electronic temperature is normally raised higher than $T_{\mathrm{c}}$. Micromagnetics based on the LLG equation cannot work under these circumstances while micromagnetics based on the LLB equation has proved to describe correctly the observed fs magnetization dynamics. ${ }^{11,12}$ An important property of the LLB theory, the longitudinal relaxation of the magnetization magnitude, appears to be responsible for the novel demagnetization mechanism and the linear reversal path. ${ }^{12,13}$ Another exciting area of magnetism where the LLB micromagnetics can be put at work are the spin-torque effect with Joule heating ${ }^{14}$ or spin-based caloric effects, such as thermally driven domain wall motion via the spin Seebeck effect. ${ }^{15}$ Important industrial applications for LLB micromagnetics also include the modeling of thermally assisted MRAM ${ }^{16}$ and heat-assisted magnetic recording, one of the future possibilities to overcome the superparamagnetic limit in magnetic recording technology. ${ }^{17}$

As mentioned above, the LLB equation describes an averaged magnetization trajectory. However, at high temperatures the dispersion of individual trajectories is important; for example, when the magnetization is quenched it should describe the loss of magnetization correlations in different sites of the sample. In the laser-induced dynamics this is responsible for the slowing down of the magnetization recovery at high laser fluency as the system temperature decreases. ${ }^{9,11}$ Therefore, under these circumstances the use of the stochastic LLB equation is paramount.

It is widely known that there is no unique formalism for the introduction of the stochastic terms into a deterministic equation. For example, in the LLG equation the random field could be introduced to both precessional and damping terms or to the precessional term only or to the damping term only. ${ }^{18-20,25}$ For the LLG equation all these formulations lead to the same FP equation but with different resulting strengths of the fluctuating fields. Moreover, the stochastic terms can also be introduced as additive noise in the form of fluctuating torques. ${ }^{19,25}$ This approach also coincides with the fluctuating fields approach near the equilibrium. ${ }^{25}$ 
Garanin and Chubykalo-Fesenko ${ }^{21}$ have suggested treating the LLB equation following Brown's treatment of the LLG equation. They derived a form of the stochastic LLB equation where, similar to the LLG equation, the stochastic terms were introduced as additional formal stochastic fields, different for longitudinal and transverse fluctuations. They also introduced the FP equation and showed that the longitudinal fluctuations result in an additional decrease of the switching time of magnetic nanoparticles at elevated temperatures. However, as we show in the present paper, although near equilibrium the resulting stochastic equation is consistent with the FDT, the requirement of the Boltzmann distribution in equilibrium is not fulfilled in the vicinity of the Curie temperature. This is in contrast to the stochastic LLG equation where both approaches are in complete agreement. In the present paper we introduce a different form of the stochastic LLB equation, consistent with the Boltzmann distribution at arbitrary temperature.

\section{TWO FORMS OF THE STOCHASTIC LLB EQUATION}

Applying a general statistical mechanics approach to the LLG equation, Brown has suggested introducing the stochastic terms into a deterministic equation of motion as "formal concepts, introduced for convenience, to produce the fluctuations $\delta M$ "; see Ref. 2. Thus these terms are not based on a kind of "first principles" approach, although some attempts to justify their final form exist in the literature and show that their properties (such as the absence of memory effects) are valid with some special assumptions only. ${ }^{22,23}$

Since the fluctuating variables are formal, their choice is multiple. For the LLG equations it is customary to introduce three-component noise variable in the form of the fluctuating field in the precessional or damping or both terms. Stochastic variables can also be introduced as additive noise in the form of fluctuating torques. ${ }^{19,25}$ This approach also coincides with the fluctuating fields one if the linearization of the LLG equation is performed. ${ }^{25}$ All these formulations lead to the same FP equation but with different resulting strengths of the fluctuating variables. For the LLB equation, however, it has been shown ${ }^{21,25}$ that the naive introduction of the same fluctuating field in all terms (precessional, longitudinal relaxation, and transverse relaxation) and the application of the FDT does not lead to convenient properties. The fluctuating fields should have correlations, not only between different spin sites but between different components $(x, y, z)$ as well.

The stochastic LLB equations which we study in the present paper have been designed to fulfill the properties of the simplest noise variables to be isotropic and uncorrelated in sites and components. The stochastic LLB equation, introduced in Ref. 21 (called here sLLB-I) is written for each macrospin (nanoparticle or discretization element) describing its average spin polarization $\mathbf{m}=\mathbf{M} / M_{\mathrm{s}}^{0}\left(\mathbf{M}\right.$ is the magnetization and $M_{\mathrm{s}}^{0}$ is the saturation magnetization value at $T=0$ ) in the following form:

$$
\begin{aligned}
\dot{\mathbf{m}}= & \gamma\left[\mathbf{m} \times \mathbf{H}_{\mathrm{eff}}\right]+\frac{|\gamma| \alpha_{\|}}{m^{2}}\left(\mathbf{m} \cdot\left(\mathbf{H}_{\mathrm{eff}}+\zeta_{\|}\right)\right) \mathbf{m} \\
& -\frac{|\gamma| \alpha_{\perp}}{m^{2}}\left[\mathbf{m} \times\left[\mathbf{m} \times\left(\mathbf{H}_{\mathrm{eff}}+\zeta_{\perp}\right)\right]\right],
\end{aligned}
$$

where $\gamma$ is the gyromagnetic ratio, $\alpha_{\|}$and $\alpha_{\perp}$ are dimensionless longitudinal and transverse damping parameters given by

$$
\alpha_{\|}=\lambda \frac{2 T}{3 T_{\mathrm{c}}}, \quad \alpha_{\perp}=\lambda\left[1-\frac{T}{3 T_{\mathrm{c}}}\right], \quad T<T_{\mathrm{c}},
$$

and $T_{\mathrm{c}}$ is the Curie temperature. For $T>T_{\mathrm{c}}, \alpha_{\perp}$ equals $\alpha_{\|}$. Here $\lambda$ is the parameter describing the coupling of the spins to the heat bath on an atomistic level. The effective field $\mathbf{H}_{\mathrm{eff}}$ is given by

$$
\mathbf{H}_{\mathrm{eff}}=\mathbf{H}+\mathbf{H}_{A}+ \begin{cases}\frac{1}{2 \tilde{\chi}_{\|}}\left(1-\frac{m^{2}}{m_{\mathrm{e}}^{2}}\right) \mathbf{m}, & T \lesssim T_{\mathrm{c}}, \\ -\frac{1}{\tilde{\chi} \|}\left(1+\frac{3}{5} \frac{T_{\mathrm{c}}}{T-T_{\mathrm{c}}} m^{2}\right) \mathbf{m}, & T \gtrsim T_{\mathrm{c}} .\end{cases}
$$

Here $m_{\mathrm{e}}$ is the zero-field equilibrium spin polarization for a given temperature. $\mathbf{H}$ and $\mathbf{H}_{A}$ are applied and anisotropy fields, respectively; $\tilde{\chi}_{\|}=(d m / d H)_{H \rightarrow 0}$ is the the longitudinal susceptibility. Note that for simplicity we present the classical version of the LLB equation; for a finite spin value see Ref. 26.

Perpendicular and longitudinal noise parameters have the following properties:

$$
\left\langle\zeta^{\mu}\right\rangle=0, \quad\left\langle\zeta_{i}^{\mu}(0) \zeta_{j}^{\nu}(t)\right\rangle=\frac{2 k_{\mathrm{B}} T}{|\gamma| M_{\mathrm{s}}^{0} V \alpha_{\mu}} \delta_{i j} \delta_{\mu \nu} \delta(t),
$$

where $\mu, v=\|, \perp$ and indices $i, j$ denote components $x, y, z$ and $V$ is the particle volume.

In this equation the formal stochastic variables were introduced as additional random fields. The noise is isotropic and multiplicative and for an individual spin it has six dimensions; i.e., the random fields acting on longitudinal and transverse damping have different properties. No random field was assumed in the precessional term. From the point of view of the FDT, this avoids the presence of correlations between different field components and different spins. This form of the stochastic LLB has been used in previous publications. . $^{9}, 10,12,21$ Here we demonstrate that this approach is not completely satisfactory in that, at elevated temperatures, the Boltzmann distribution is not correctly recovered. In order to explore this inconsistency we propose an alternative approach, introducing instead of an additional random field, an additional random torque in the longitudinal direction in an additive manner, the choice of which we justify in Sec. III. This leads to a different stochastic differential equation, called here sLLB-II, which we propose in this paper:

$$
\begin{aligned}
\dot{\mathbf{m}}= & \gamma\left[\mathbf{m} \times \mathbf{H}_{\mathrm{eff}}\right]+\frac{|\gamma| \alpha_{\|}}{m^{2}}\left(\mathbf{m} \cdot \mathbf{H}_{\mathrm{eff}}\right) \mathbf{m} \\
& -\frac{|\gamma| \alpha_{\perp}}{m^{2}}\left[\mathbf{m} \times\left[\mathbf{m} \times\left(\mathbf{H}_{\mathrm{eff}}+\boldsymbol{\eta}_{\perp}\right)\right]\right]+\boldsymbol{\eta}_{\|} .
\end{aligned}
$$

In what follows we apply to this equation both the FDT and the FP equation approaches. Both approaches give the following properties of the fluctuating terms:

$$
\begin{aligned}
& \left\langle\eta_{i}^{\mu}\right\rangle=0, \quad\left\langle\eta_{i}^{\perp}(0) \eta_{j}^{\perp}(t)\right\rangle=\frac{2 k_{\mathrm{B}} T\left(\alpha_{\perp}-\alpha_{\|}\right)}{|\gamma| M_{\mathrm{s}}^{0} V \alpha_{\perp}^{2}} \delta_{i j} \delta(t), \\
& \left\langle\eta_{i}^{\|}(0) \eta_{j}^{\|}(t)\right\rangle=\frac{2|\gamma| k_{\mathrm{B}} T \alpha_{\|}}{M_{\mathrm{s}}^{0} V} \delta_{i j} \delta(t), \quad\left\langle\eta_{i}^{\|} \eta_{j}^{\perp}\right\rangle=0 .
\end{aligned}
$$


The noise components are again isotropic. Note that $\alpha_{\perp}>\alpha_{\|}$, according to Eq. (2), and that in this noise representation the transverse fluctuations are absent above $T_{\mathrm{c}}$, where $\alpha_{\perp}=\alpha_{\|}$.

Note that in the present paper Eqs. (1) and (5) are interpreted as Stratonovich stochastic differential equations and the fluctuating noise variables (4) and (6) as the time derivatives of scalar Wiener processes.

The application of the FDT (see Appendix A) justifies that for both Eqs. (1) and (5), the particular choices of stochastic variables provide isotropic and uncorrelated (in different spin sites and components) noise properties near equilibrium. In the next section and based on the same choice of the stochastic variables, the FP equations are derived for both Langevin equations. As we will see, only for Eq. (5) the equilibrium distribution is the Boltzmann one in the whole temperature range. Thus, this equation is recommended as the only valid approach at temperatures around $T_{c}$. This is especially important for simulation of heat-assisted magnetic recording.

\section{THE FOKKER-PLANCK EQUATION}

Once a convenient set of isotropic and uncorrelated noise variables has been found, the derivation of the FP equation from the known stochastic equation of motion is straightforward. ${ }^{28}$ As a first step, we will do this for noninteracting systems only. Consider a general system of differential equations in the Stratonovich form with multiplicative noise

$$
d m_{k}=a_{k} d t+b_{k j} \circ \Delta W_{j} .
$$

Here the subscript $k$ denotes stochastic variables, $j$ numbers independent standard Wiener processes $\triangle W_{j}$, and the sign $\circ$ indicates the Stratonovich calculus. For stochastic LLB equations we have vector equations for the variable $\mathbf{m}$ and the vector $a$ stands for the deterministic part, common for both sLLB-I and sLLB-II:

$$
\begin{aligned}
a(\mathbf{m}, t)= & \gamma\left[\mathbf{m} \times \mathbf{H}_{\mathrm{eff}}\right]+\frac{|\gamma| \alpha_{||}}{m^{2}}\left(\mathbf{m} \cdot \mathbf{H}_{\mathrm{eff}}\right) \mathbf{m} \\
& -\frac{|\gamma| \alpha_{\perp}}{m^{2}}\left[\mathbf{m} \times\left[\mathbf{m} \times\left(\mathbf{H}_{\mathrm{eff}}\right)\right]\right],
\end{aligned}
$$

while the matrix $b_{k j}$ is defined by the stochastic part, different for sLLB-I and SLLB-II. Note that for the one-spin LLB equation the index $k$ stands for spin components $x, y, z$ and there are 6 independent Wiener processes.

The FP equation gives access to the dynamics of the probability function $f(\mathbf{m}, t)$, which is defined as the probability to find the average magnetic moment at position $\mathbf{m}$ and time $t$. The form of the FP equation is completely defined by the stochastic differential Eq. (7) and requires calculations of the diffusion and drift terms.. The FP equation reads

$$
\frac{\partial f}{\partial t}=-\sum_{i} \frac{\partial\left(a_{i} f\right)}{\partial m_{i}}+\frac{1}{2} \sum_{i, j, k} \frac{\partial}{\partial m_{i}}\left\{b_{i k} \frac{\partial}{\partial m_{j}}\left[b_{j k} f\right]\right\},
$$

where the first and second terms are the drift and diffusive terms respectively.

We assume for the sLLB-I Eq. (1) the properties of Eq. (4), i.e., the isotropic form and absence of correlations between components with unknown at this stage magnitude of the correlators

$$
\left\langle\zeta^{\nu}(t) \zeta^{\nu}(0)\right\rangle=2 D_{\nu} \delta(t)
$$

where $v=\|, \perp$ and the indices corresponding to different spin components $x, y, z$ are omitted for simplicity. Here $2 D_{\|}, 2 D_{\perp}$ are diffusion coefficients to be defined from the thermodynamic equilibrium. For stochastic LLB equations, we denote 6 independent noise variables with $x, y, z$ and perpendicular $\perp$ and parallel || symbols, corresponding to different components of magnetization and parallel or perpendicular damping terms. The elements of the $b_{k j}$ matrix, corresponding to parallel || and perpendicular $\perp$ noise variables, are

$$
\begin{aligned}
& b_{k j}^{\|}=\frac{\alpha_{\|}|\gamma| \sqrt{2 D_{\|}}}{m^{2}} m_{k} m_{j}, \\
& b_{k j}^{\perp}=\alpha_{\perp}|\gamma| \sqrt{2 D_{\perp}}\left(-\frac{m_{k} m_{j}}{m^{2}}+\delta_{k j}\right) .
\end{aligned}
$$

Here both $k$ and $j$ indices correspond to components $x, y, z$.

Substituting these into the FP equation (9), calculating the derivatives, and collecting all the terms, one comes to the following FP equation:

$$
\begin{aligned}
\frac{\partial f}{\partial t}= & -\frac{\partial}{\partial \mathbf{m}}\left\{\gamma\left[\mathbf{m} \times \mathbf{H}_{\mathrm{eff}}\right] f+\frac{\alpha_{\|}|\gamma|}{m^{2}}\left(\mathbf{m} \cdot \mathbf{H}_{\mathrm{eff}}\right) \mathbf{m} f-\frac{\alpha_{\perp}|\gamma|}{m^{2}}\left[\mathbf{m} \times\left[\mathbf{m} \times \mathbf{H}_{\mathrm{eff}}\right]\right] f\right\} \\
& -\frac{\partial}{\partial \mathbf{m}}\left\{\frac{D_{\perp} \alpha_{\perp}^{2}|\gamma|^{2}}{m^{2}}\left[\mathbf{m} \times\left[\mathbf{m} \times \frac{\partial f}{\partial \mathbf{m}}\right]\right]-\frac{D_{\|} \alpha_{\|}^{2}|\gamma|^{2}}{m^{2}} \mathbf{m}\left(\mathbf{m} \cdot \frac{\partial f}{\partial \mathbf{m}}\right)\right\}-2 D_{\|} \alpha_{\|}^{2}|\gamma|^{2} \frac{\partial}{\partial \mathbf{m}}\left(\frac{\mathbf{m}}{m^{2}} f\right) .
\end{aligned}
$$

The FP equation above differs from the one suggested originally in Ref. 21 by the presence of the last term. Note that for small deviations from equilibrium, i.e., under conditions for which the FDT was applied (not close to $T_{\mathrm{c}}$ ), this term is zero to the first order.

The FP equation should be solved in the stationary case to find the equilibrium function from which the unknown coefficients can be defined. In the conventional statistical mechanics one normally searches for the equilibrium solution in the form of the Boltzmann distribution:

$$
\begin{gathered}
f=f_{0} \exp \left(-F(\mathbf{m}) / k_{\mathrm{B}} T\right), \\
\frac{\partial f}{\partial \mathbf{m}}=\frac{V M_{\mathrm{s}}^{0}}{k_{\mathrm{B}} T} \mathbf{H}_{\mathrm{eff}} f .
\end{gathered}
$$

where $F$ is the free energy.

Here $f_{0}$ is a normalization constant. It is easy to see that the FP equation (12) in equilibrium cannot be satisfied with 
the Boltzmann distribution function. After the substitution $f=\bar{f} / m^{2}$, it is possible to show that in equilibrium the function $\bar{f}$ is the Boltzmann distribution (13). This allows us to calculate the magnitudes of the correlators (10) obtaining the properties indicated in Eq. (4). Thus the equilibrium solution of the FP equation (12) is not the Boltzmann distribution but

$$
f=\tilde{f}_{0} \frac{1}{m^{2}} \exp \left(-F / k_{\mathrm{B}} T\right)
$$

(here $\tilde{f}_{0}$ is a new normalization constant), as indeed we will see in the numerical simulations in Sec. IV.

The sLLB-II equation (5) was constructed in order to satisfy the FP equation derived in Ref. 21 and to correct for the additional drift term. Provided that $\left\langle\eta^{\nu}(t) \eta^{\nu}(0)\right\rangle=2 \tilde{D}_{\nu} \delta(t)$, $v=\|, \perp$, for this equation we have

$$
b_{k j}^{\|}=\sqrt{2 \tilde{D}_{\|}} \delta_{k j}
$$

and the same values for the perpendicular part as in Eq. (11) (with $D_{\perp} \rightarrow \tilde{D}_{\perp}$ ). The corresponding FP equation reads

$$
\begin{aligned}
\frac{\partial f}{\partial t}= & -\frac{\partial}{\partial \mathbf{m}}\left\{\gamma\left[\mathbf{m} \times \mathbf{H}_{\mathrm{eff}}\right] f+\frac{\alpha_{\|}|\gamma|}{m^{2}}\left(\mathbf{m} \cdot \mathbf{H}_{\mathrm{eff}}\right) \mathbf{m} f\right. \\
& \left.-\frac{\alpha_{\perp}|\gamma|}{m^{2}}\left[\mathbf{m} \times\left[\mathbf{m} \times \mathbf{H}_{\mathrm{eff}}\right]\right] f\right\} \\
& -\frac{\partial}{\partial \mathbf{m}}\left\{\frac{\tilde{D}_{\perp} \alpha_{\perp}^{2}|\gamma|^{2}}{m^{2}}\left[\mathbf{m} \times\left[\mathbf{m} \times \frac{\partial f}{\partial \mathbf{m}}\right]\right]-\tilde{D}_{\|} \frac{\partial f}{\partial \mathbf{m}}\right\} .
\end{aligned}
$$

Using the relation (14), it is easy to check that in equilibrium this equation has a solution of the Boltzmann distribution function (13). This provides the following conditions for the fluctuating strength properties:

$$
\begin{array}{r}
\alpha_{\|}-\alpha_{\perp}+\frac{\tilde{D}_{\perp} \alpha_{\perp}^{2}|\gamma| V M_{\mathrm{s}}^{0}}{k_{\mathrm{B}} T}=0, \\
\alpha_{\perp}-\frac{\tilde{D}_{\perp} \alpha_{\perp}^{2}|\gamma| V M_{\mathrm{s}}^{0}}{k_{\mathrm{B}} T}-\frac{\tilde{D}_{\|} V M_{\mathrm{s}}^{0}}{k_{\mathrm{B}} T|\gamma|}=0,
\end{array}
$$

from which Eqs. (6) are deduced.

Using the relation

$$
m^{2} \frac{\partial f}{\partial \mathbf{m}}=-\left[\mathbf{m} \times\left[\mathbf{m} \times \frac{\partial f}{\partial \mathbf{m}}\right]\right]+\left(\mathbf{m} \cdot \frac{\partial f}{\partial \mathbf{m}}\right) \mathbf{m},
$$

we can finally cast the FP equation (17) in a conventional form which coincides with the one presented in Ref. 21:

$$
\frac{\partial f}{\partial t}+\frac{\partial}{\partial \mathbf{m}} \mathbf{J}=0
$$

where the probability current $\mathbf{J}$ is given by

$$
\begin{aligned}
\mathbf{J}= & \gamma\left[\mathbf{m} \times f \mathbf{H}_{\mathrm{eff}}\right]+\alpha_{\|}|\gamma| \frac{\mathbf{m}}{m^{2}}\left[\mathbf{m} \cdot\left(f \mathbf{H}_{\mathrm{eff}}-\frac{k_{\mathrm{B}} T}{M_{\mathrm{s}}^{0} V} \frac{\partial f}{\partial \mathbf{m}}\right)\right] \\
& -\frac{\alpha_{\perp}|\gamma|}{m^{2}}\left[\mathbf{m} \times\left[\mathbf{m} \times\left(f \mathbf{H}_{\mathrm{eff}}-\frac{k_{\mathrm{B}} T}{M_{\mathrm{s}}^{0} V} \frac{\partial f}{\partial \mathbf{m}}\right)\right]\right] .
\end{aligned}
$$

\section{NUMERICAL TESTS}

In order to compare the properties of the two different forms of the LLB equation, we have implemented the stochastic equation in both forms, using the Stratonovich interpretation and the Heun numerical scheme. ${ }^{18}$ Some useful properties of the stochastic equations are summarized in Appendix B. The Heun numerical scheme is of the predictor-corrector type and is especially convenient since the correction to the drift due to the influence of the predictor coincides exactly with the Ito-Stratonovich drift. Thus in the Stratonovich interpretation the scheme is the same as for the deterministic equation.

For the LLB equation, the free energy of the system is conveniently defined as ${ }^{6,9}$

$$
\frac{F}{M_{\mathrm{s}}^{0} V}= \begin{cases}\frac{m_{x}^{2}+m_{y}^{2}}{2 \tilde{\chi}_{\perp}}+\frac{\left(m^{2}-m_{\mathrm{e}}^{2}\right)^{2}}{8 \tilde{\chi}_{\|} m_{\mathrm{e}}^{2}}, & T \leqslant T_{\mathrm{c}}, \\ \frac{m_{x}^{2}+m_{y}^{2}}{2 \tilde{\chi}_{\perp}}+\frac{3}{20 \tilde{\chi}_{\|}} \frac{T_{\mathrm{c}}}{T-T_{\mathrm{c}}}\left(m^{2}+\frac{5}{3} \frac{T-T_{\mathrm{c}}}{T_{\mathrm{c}}}\right)^{2}, & T>T_{\mathrm{c}} .\end{cases}
$$

Here the longitudinal and the perpendicular susceptibilities $\tilde{\chi}_{\|}$, $\tilde{\chi}_{\perp}$ and the equilibrium magnetization $m_{\mathrm{e}}$ are all temperaturedependent values. The first term provides a uniaxial anisotropy, while the second term controls the length of the magnetization. At low temperatures the second term keeps the magnitude of the vector $\mathbf{m}$ very close to $m_{\mathrm{e}}$, due to the fact that $\tilde{\chi}_{\|} \ll 1$ for all temperatures not too close to $T_{\mathrm{c}}$. In this case both sLLB-II and sLLB-I trivially give the same result coinciding with that of the LLG equation with temperature-dependent parameters. The deviations from the LLG case are defined by the parameter ${ }^{21} \tilde{\chi}_{\|} / \tilde{\chi}_{\perp}$, i.e., are better seen close to $T_{\mathrm{c}}$ when the parallel susceptibility is not small and for high-anisotropy materials such as FePt, for which $\tilde{\chi}_{\perp}$ is also small.

As an input into the single-spin LLB equation we need temperature-dependent macroscopic parameters: the magnetization $m_{\mathrm{e}}(T)$ and perpendicular and parallel susceptibilities $\tilde{\chi}_{\perp}(T)$ and $\tilde{\chi}_{\|}(T)$, respectively. These can be taken either from experiment or evaluated numerically. The easiest approximate way is to evaluate these parameters from a mean-field approach (MFA). ${ }^{10}$ In order to make the following results as realistic as possible, we have performed all calculations with parameters extracted from an atomistic Langevin dynamics model of FePt parameterized with density functional theory calculations, a comprehensive description of which can be found in Ref. 9. This latter approach is more likely to form the basis of future applications for the LLB, due to the specificity of the parameters to the problem of interest, and the multiscale approach to the problem enabling the incorporation of a significant level of details into the macroscopic model.

The following calculations utilize the FePt input parameters from Ref. 9, which in summary give magnetic characteristics of $T_{\mathrm{c}}$ of $660 \mathrm{~K}$ and magnetocrystalline anisotropy energy density of $7.7 \times 10^{6} \mathrm{~J} / \mathrm{m}^{3}$. The high anisotropy accentuates the differences between the sLLB-I and sLLB-II, and this is especially true near $T_{\mathrm{c}}$, where any deviations from the expected Boltzmann distribution become obvious. For the integration we use a time step with $\Delta t=1 \mathrm{fs}$. The intrinsic damping is set to $\lambda=1.0$, since we are interested in equilibrium distributions. The gyromagnetic ratio has the usual value of $\gamma=1.76 \times$ $10^{11} \mathrm{~T}^{-1} \mathrm{~s}^{-1}$.

The first case of interest is the distribution in the length of magnetization, $P(|m|)$. The presence of anisotropy results in symmetry breaking and gives rise to a 3D free-energy surface. Therefore for simplicity the following results are calculated 


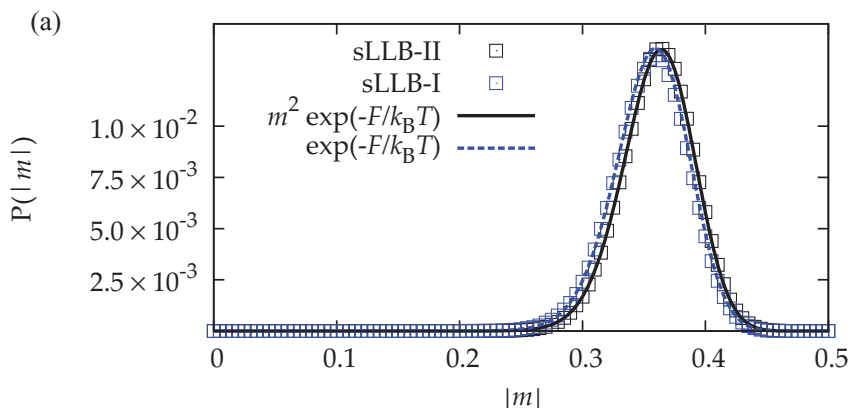

(b)

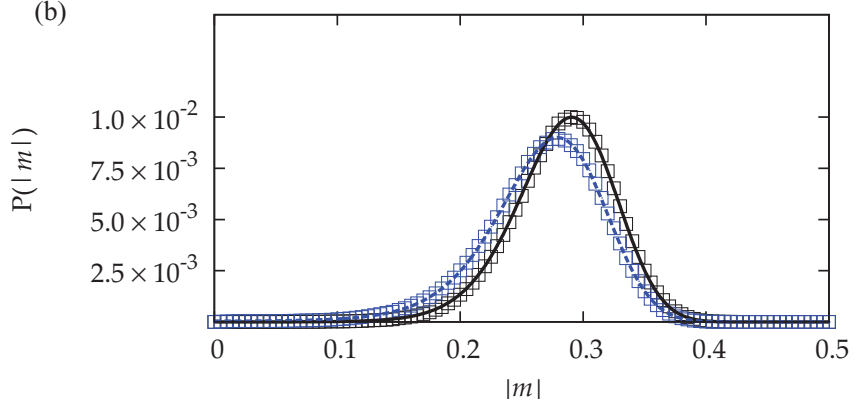

(c)

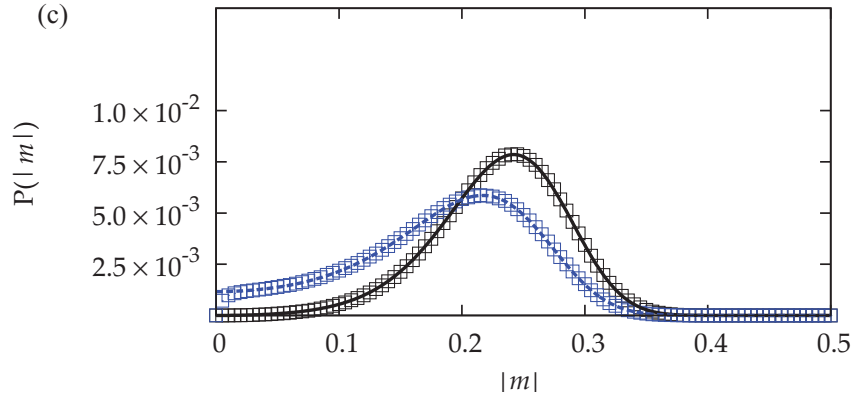

FIG. 1. (Color online) Isotropic distributions of the magnitude of the magnetization, comparing the sLLB-II and sLLB-I at (a) $T=$ $620 \mathrm{~K}$, (b) $T=640 \mathrm{~K}$, and (c) $T=650 \mathrm{~K}$.

for the isotropic case, with $\chi_{\perp}=\infty$. The system size was set so that $M_{\mathrm{s}}^{0} V=1.5 \times 10^{-19} \mathrm{~J} \mathrm{~T}^{-1}$, equivalent to a cell size of $5.3 \mathrm{~nm}$. The system is first equilibrated for $1 \mathrm{~ns}\left(10^{6}\right.$ time steps $)$ and then the distribution is sampled over $10 \mathrm{~ns}\left(10^{7}\right.$ time steps). The isotropic distributions for the sLLB-I and sLLB-II equation are plotted in Fig. 1.

For temperatures not close to $T_{\mathrm{c}}$ the two forms of the LLB equation possess nearly identical distributions, barely distinguishable from the noise and coinciding with the Boltzmann form, as is evident from the data for $T=620 \mathrm{~K}$. As the temperature becomes closer to $T_{\mathrm{c}}$ and the value of $m_{\mathrm{e}}$ becomes small, the distributions show significant differences. At the higher temperature of $T=650 \mathrm{~K}$, the sLLB-II fits perfectly with the expected Boltzmann distribution

$$
P(|m|) \propto m^{2} \exp \left(-\frac{F}{k_{\mathrm{B}} T}\right),
$$

corresponding to the distribution Eq. (13) for an axially symmetric case. The results from sLLB-I fits with the anticipated (non-Boltzmann) distribution Eq. (15), i.e., without the $m^{2}$ factor in Eq. (22).

Next we consider the anisotropic case, where the distributions are different along different axes. In order to assess the raw distribution, it is necessary to record the probabilities for all $m_{x}, m_{y}$, and $m_{z}$ in the unit sphere. This way the phase-space volume is equal for all elements, and it is possible to take "slices" along certain interesting directions, such as $m_{y} \approx 0$, and compare that with the analytic distribution directly. The presence of anisotropy gives the magnetization a preferential direction along the $z$ axis. Probability distributions along $m_{z}$ and $m_{x}$ for different temperatures are plotted in Fig. 2 and they are in agreement with the corresponding analytical distributions.

As before, the plots show near perfect agreement of the distributions at $620 \mathrm{~K}$. At $T=640 \mathrm{~K}$ we note the presence of a peak for $|m|=0$ for the sLLB-I due to the $1 / \mathrm{m}^{2}$ factor in the corresponding distribution (15). Differences in the distributions for sLLB-I and sLLB-II become apparent as the temperature approaches $T_{\mathrm{c}}$. Importantly, sLLB-II gives the Boltzmann distribution (13) even at elevated temperatures and as such is the form to be used close to $T_{\mathrm{c}}$.

Given the differences in the equilibrium distributions for the two stochastic forms of the LLB equation, it is important to ascertain the differences in terms of the overall behavior of the system. As our next step we consider the behavior of the average magnetization of an ensemble of noninteracting spins as a function of temperature (see Fig. 3). The average magnetization length is defined as thermal average

$$
|m|=\sqrt{\left\langle m_{x}^{2}\right\rangle+\left\langle m_{y}^{2}\right\rangle+\left\langle m_{z}^{2}\right\rangle}
$$

with

$$
\left\langle m_{x}^{2}\right\rangle=\frac{1}{t} \int_{0}^{t} m_{x}^{2}\left(t^{\prime}\right) d t^{\prime}
$$

and similar definitions for $\left\langle m_{y}^{2}\right\rangle$ and $\left\langle m_{z}^{2}\right\rangle$.

The results are presented for different system sizes showing important size effects and deviations close to $T_{\mathrm{c}}$. The results converge for larger system sizes due to the fact that the LLB equation has been derived in the MFA model, i.e., effectively for the infinite system. As can be seen both approaches sLLB-II and sLLB-I give practically indistinguishable results almost for the total temperature range, being the average value provided by the sLLB-I always slightly to the left with respect to SLLB-II. Note that it can be shown analytically that both distributions (13) and (15) displace an initial value $m_{\mathrm{e}}$ relative to the input one. The additional shift depends on the longitudinal susceptibility and is slightly larger for sLLB-II than for sLLB-I. Thus, the effect should be visible for large temperatures only where it competes with finite-size effects. The fact that the average magnetization value differs from the input one constitutes a real problem for the multiscale modeling technique for which the conservation of the average magnetization length, calculated on the basis of the lower rank approach, is desirable. This problem will be addressed in the future.

\section{CONCLUSIONS AND DISCUSSION}

In the present paper we have introduced a stochastic form of the LLB equation which is consistent with the Boltzmann distribution function in the whole temperature range, including very close to the Curie temperature, called here sLLB-II. 

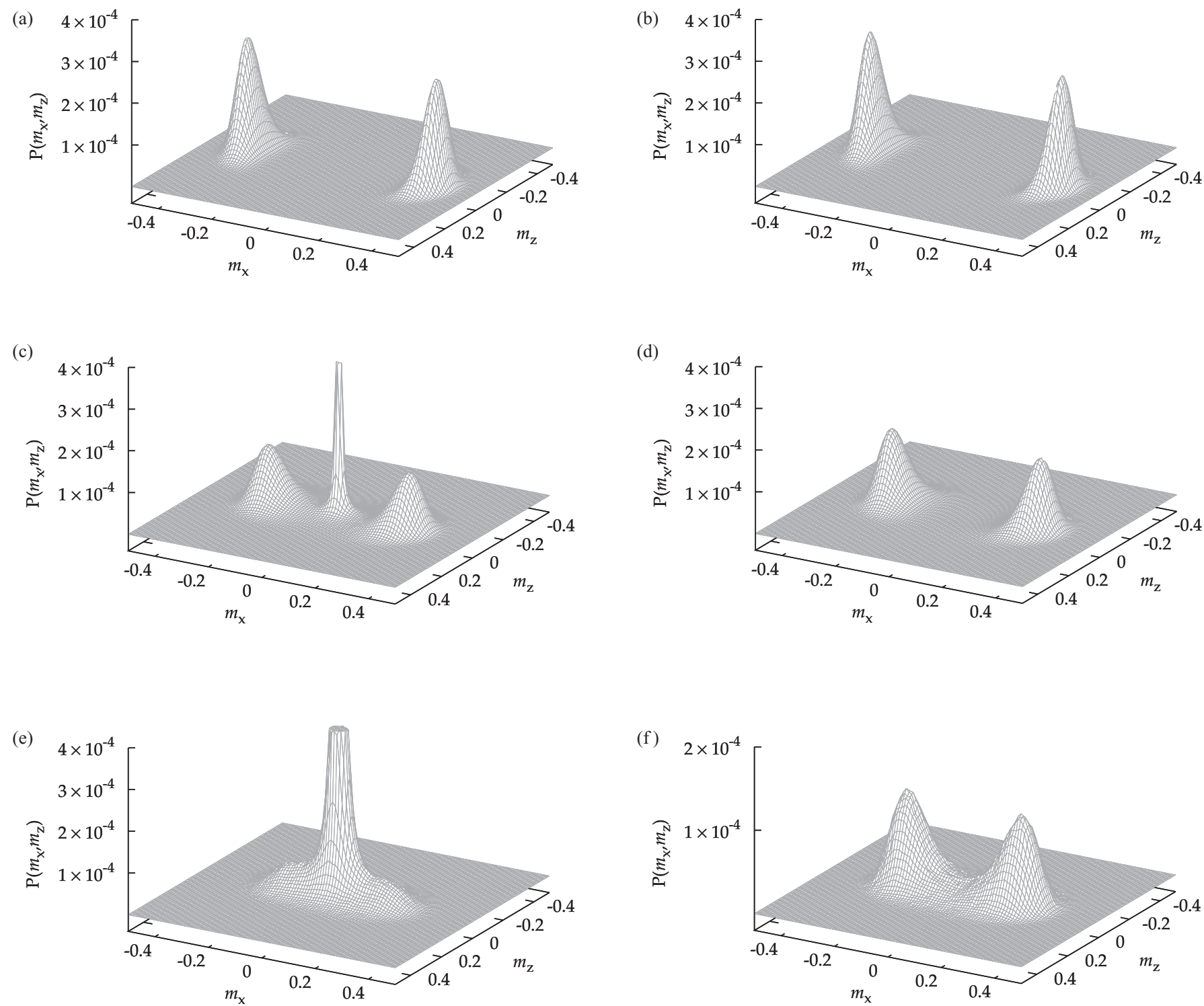

FIG. 2. Anisotropic distributions of magnetization slices for $m_{y} \approx 0$, comparing the sLLB-I (left) and sLLB-II (right) at [(a), (b)] $T=620 \mathrm{~K}$, [(c), (d)] $T=640 \mathrm{~K}$, and [(e), (f)] $T=650 \mathrm{~K}$.

Unlike the previously introduced stochastic LLB equation (sLLB-I), where both fluctuating fields were introduced as a multiplicative noise, ${ }^{21}$ in the sLLB-II equation the longitudinal fluctuations are introduced as an additive stochastic process. We have proved the properties of the stochastic variables for the sLLB-II basing on the FDT as well as on the FP equation approaches. Unlike the case of the LLG equation where the two approaches gave the same result, the sLLB-I equation appeared to be consistent with the FDT approach but, contrary to the sLLB-II equation, not consistent with the Boltzmann distribution at high temperatures.

The results were checked in numerical simulations. Although detailed distribution functions close to the Curie temperatures appeared to be very different, at the present moment we did not see prominent differences in the evaluation of the macroscopic properties such as average magnetization, the switching time ${ }^{21}$ or the ultrafast magnetization dynamics. ${ }^{12}$
Examples showing significant differences in the physical behavior of macroscopic properties are yet to be found.

The detailed numerical simulations also show some further problems in the use of the stochastic LLB equation within the multiscale description. Due to the approximation in the derivation of the free energy Eq. (21), the calculation of average magnetization using this particular functional form does not reproduce exactly the input value of the average magnetization $m_{\mathrm{e}}$, although it is close to it for large system sizes. The latter should be considered as a necessary condition in the multiscale description. If the free energy were evaluated on the basis of the full atomistic description, the correct magnetization value would be recovered. Furthermore, we should also notice the presence of finite-size effects, although not so pronounced as in the modeling using the LLG approach. The corrections of these problems will be the subject of further investigation. 

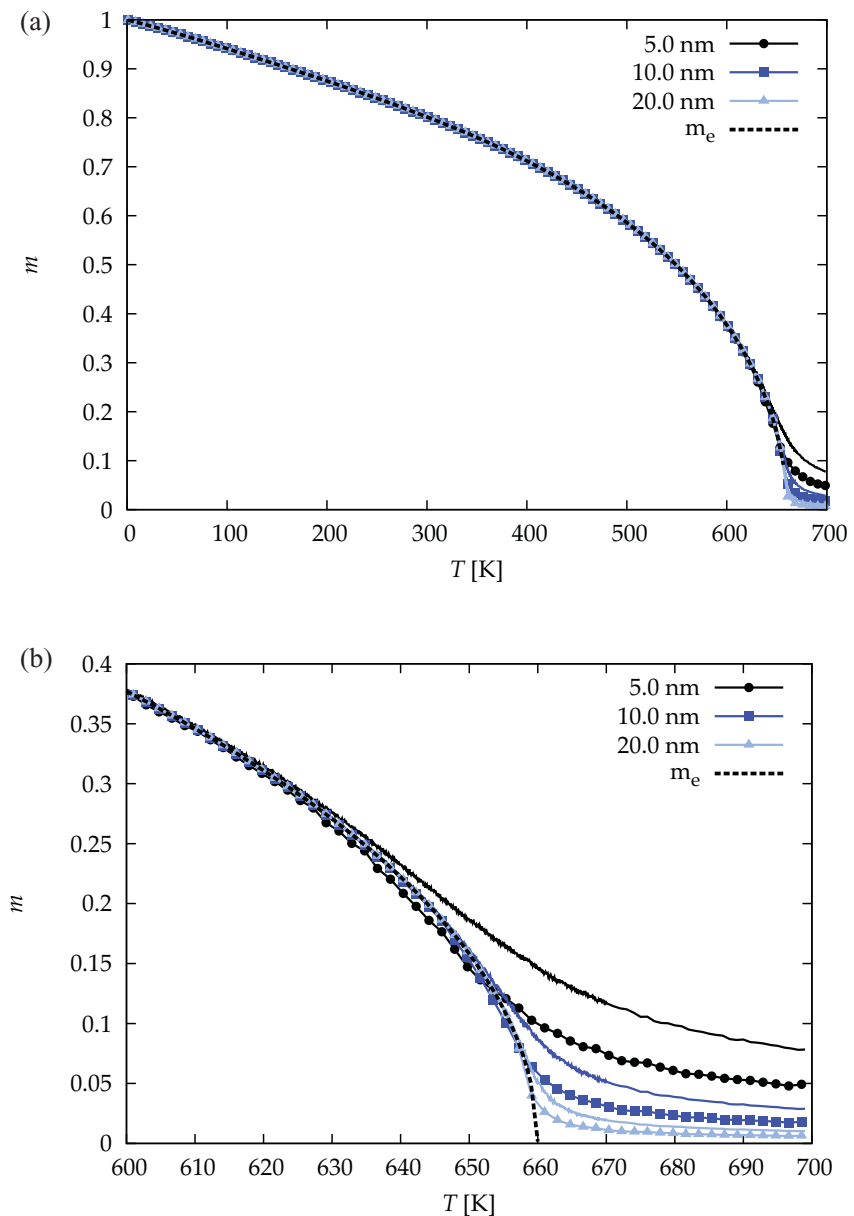

FIG. 3. (Color online) Magnetization versus temperature (a) for the whole temperature range and (b) close to the Curie temperature $T_{\mathrm{c}}$, for different system sizes. The results provided by the sLLB-II (lines) are always to the right with respect to those calculated within the sLLB-I (symbols). The black line represents an input $m_{\mathrm{e}}$ value.

\section{ACKNOWLEDGMENTS}

The authors are grateful to Pierre Asselin for pointing out this problem and for helpful discussions. D.H. and U.N. acknowledge financial support from the Deutsche Forschungsgemeinschaft via Sonderforschungsbereich 767. U.A. and O.C.-F. gratefully acknowledge funding from the Spanish Ministry of Science and Innovation under Grant No. FIS2010-20979-C02-02. Support of the EU FP7 program [Grants No. NMP3-SL-2008-214469 (UltraMagnetron) and No. 214810 (FANTOMAS)] is also gratefully acknowledged.

\section{APPENDIX A: THE FLUCTUATION-DISSIPATION THEOREM}

The approach based on the FP equation has the great advantage to deal with a complete nonlinear problem resulting from the multiplicative noise in terms of the probability distribution functions. ${ }^{18}$ The approach based on the FDT, ${ }^{24,25}$ however, is a linear-response theory, valid for small deviations from the equilibrium only. One may wonder, therefore, why the FDT is necessary at all, since it is valid for linearized systems only. The problem is that one should provide for the FP equation the choice of convenient stochastic variables which could potentially lead to a correct equilibrium with the desired properties of being isotropic and uncorrelated in space and time. However, not all assumptions of the formal stochastic variables are consistent with this requirement. The FDT approach in this sense is more general than FP. Given a formal assumption for the fluctuating variables, it allows proving their equilibrium properties based on the Onsager relation. This allows choosing convenient fluctuating variables with desired properties of being isotropic and uncorrelated.

Generally speaking, the noise in Eqs. (1) and (5) is multiplicative. However, close to equilibrium, one can linearize the stochastic equations, converting the noise variables to additive. Below we use a standard close-to-equilibrium statistical mechanics based on the Onsager reciprocal relations close to equilibrium. This approach can be applied for a multispin case without any additional difficulty so that the indices $i$ and $j$ in this subsection refer to both spin components and different sites.

Following Refs. 24, 25 and 27, let us consider a general form of the linear Langevin equation of motion for thermodynamically conjugate variables $x_{i}$ and $X_{i}=\left(1 / k_{\mathrm{B}} T\right)\left(\partial F / \partial x_{i}\right)$ :

$$
\dot{x_{i}}=-\sum_{j} \gamma_{i j} X_{j}+f_{i},
$$

where $F$ is the free energy, $\gamma_{i j}$ are the so-called kinetic coefficients, and $f_{i}$ are the components of a random force representing thermal fluctuations in the system and the indices $i$ and $j$ denote the particle's degrees of freedom. According to the FDT in the Onsager formulation, the properties of $f_{i}$ are related to $\gamma_{i j}$ as follows:

$$
\left\langle f_{i}(t)\right\rangle=0, \quad\left\langle f_{i}(t) f_{j}\left(t^{\prime}\right)\right\rangle=\kappa_{i j} \delta\left(t-t^{\prime}\right) .
$$

Here the matrix $\kappa_{i j}=\gamma_{i j}+\gamma_{j i}$ is symmetrized.

Close to the equilibrium state, the LLB equation can be linearized using small deviations from the equilibrium values

$$
h^{i}=H_{\mathrm{eff}}^{i}-H_{\mathrm{eff}}^{i,(0)}, \quad \mu^{i}=m^{i}-m_{(0)}^{i} .
$$

For simplicity let us direct the local axis $z$ parallel to the local magnetization direction $m_{(0)}^{i}=\left(0,0, m_{0}^{i}\right)$. It is easy to show that for the LLB equation $H_{\mathrm{eff}}^{i,(0)}=0$. The consequence of the linearization of the LLB equation is that it assumes that $\left|\mu^{i}\right| \ll m_{0}^{i}$; i.e., it will not be valid near $T_{\mathrm{c}}$, where $m_{0}^{i} \approx 0$.

After linearizing, we come to the stochastic LLB equation in the form

$$
\dot{\mu}_{i}=-\sum_{j} \gamma_{i j} X_{j}=-\frac{M_{\mathrm{s}}^{0} V}{k_{\mathrm{B}} T} \sum_{j} \gamma_{i j} h_{j} ;
$$

i.e., the internal fields $h_{j}$ are linearly proportional to thermodynamically conjugated variables for $\mu_{j}$ :

$$
X_{j}=-\frac{1}{k_{\mathrm{B}} T} \frac{\partial F}{\partial \mu_{j}}=\frac{M_{\mathrm{s}}^{0} V}{k_{\mathrm{B}} T} h_{j} .
$$

The fact that the linearized LLB equation can be written exactly in the form (A4), i.e., with the magnetization as a linear combination of only the field components, is quite remarkable 
and it is this form which leads to the absence of correlations between different spin sites. It is not a general property but a consequence of the form of the damping. ${ }^{25}$ Consequently, from now on we omit indices corresponding to different spin sites referring only to different $x, y, z$ components.

The nonzero kinetic coefficients have the following forms:

$$
\begin{aligned}
& \gamma_{x x}=\gamma_{y y}=\frac{\alpha_{\perp} k_{\mathrm{B}} T|\gamma|}{M_{\mathrm{s}}^{0} V}, \quad \gamma_{z z}=\frac{\alpha_{||} k_{\mathrm{B}} T|\gamma|}{M_{\mathrm{s}}^{0} V}, \\
& \gamma_{x y}=-\gamma_{y x}=-\frac{m_{0}|\gamma| k_{\mathrm{B}} T}{M_{\mathrm{s}}^{0} V} .
\end{aligned}
$$

The reversible antisymmetric parts, coming from the precessional term, do not contribute to the thermal fluctuations after symmetrizing the kinetic coefficients for calculations of the matrix $\kappa_{i j}$ :

$$
\kappa_{x x}=\kappa_{y y}=\frac{2 \alpha_{\perp} k_{\mathrm{B}} T|\gamma|}{M_{\mathrm{s}}^{0} V}, \quad \kappa_{z z}=\frac{2 \alpha_{||} k_{\mathrm{B}} T|\gamma|}{M_{\mathrm{s}}^{0} V} .
$$

Hence we can see that the longitudinal fluctuations exist in our system and are different from the transverse ones. Finally, we can rewrite the properties of a random force $f_{i}$ as follows:

$$
\begin{gathered}
\left\langle f_{i}(t)\right\rangle=0, \quad\left\langle f_{i}(t) f_{j}\left(t^{\prime}\right)\right\rangle=0, \quad i \neq j, \\
\left\langle f_{x(y)}(t) f_{x(y)}\left(t^{\prime}\right)\right\rangle=\frac{2 \alpha_{\perp} k_{\mathrm{B}} T|\gamma|}{M_{\mathrm{s}}^{0} V} \delta\left(t-t^{\prime}\right), \\
\left\langle f_{z}(t) f_{z}\left(t^{\prime}\right)\right\rangle=\frac{2 \alpha_{\|} k_{\mathrm{B}} T|\gamma|}{M_{\mathrm{s}}^{0} V} \delta\left(t-t^{\prime}\right) .
\end{gathered}
$$

It is easy to demonstrate that in all other systems of coordinates, not coinciding with $z \| \mathbf{m}_{0}$ (the $z$ axis locally is parallel to the equilibrium magnetization direction), this noise representation leads to correlations between different noise components. Thus, the additive noise representation is inconvenient. In contrast, the linearization of the sLLB-I (1) equation leads to the following relations:

$$
\begin{aligned}
f_{x, y} & =\alpha_{\perp}|\gamma| \zeta_{x, y}^{\perp}, \\
f_{z} & =\alpha_{\|}|\gamma| \zeta_{z}^{\|},
\end{aligned}
$$

and we come finally to the following properties, coinciding with Eqs. (4):

$$
\begin{gathered}
\left\langle\zeta_{x(y), \perp}(t) \zeta_{x(y), \perp}\left(t^{\prime}\right)\right\rangle=\frac{2 k_{\mathrm{B}} T}{|\gamma| \alpha_{\perp} M_{\mathrm{s}}^{0} V} \delta\left(t-t^{\prime}\right), \\
\left\langle\zeta_{z, \|}(t) \zeta_{z, \|}\left(t^{\prime}\right)\right\rangle=\frac{2 k_{\mathrm{B}} T}{|\gamma| \alpha_{\|} M_{\mathrm{s}}^{0} V} \delta\left(t-t^{\prime}\right) .
\end{gathered}
$$

It is clear from this representation that if only one noise variable in both longitudinal and transverse damping terms $\zeta$ were used, its three components would be different due to the relations above. Transforming this to an arbitrary system of coordinates, it is easy to see that this noise is again correlated in all other systems of coordinates. However, if different noise variables are used for perpendicular and longitudinal process, then the longitudinal component of the transverse noise $\zeta_{z, \perp}$ and the transverse components of the longitudinal one $\zeta_{x(y) \|}$ remain undefined. They can be arbitrary due to the form of the LLB equation. Thus, without the loss of generality we can assume that $\zeta_{z, \|}, \zeta_{x, \|}$, and $\zeta_{y, \|}$ have the same correlators for the first and the second moments and the same for $\zeta_{z \perp}, \zeta_{x, \perp}, \zeta_{y \perp}$. This form of the noise leads to the invariance property of the noise function with respect to the change of the system of coordinates.

Similarly, for the sLLB-II Eq. (5):

$$
\begin{gathered}
f_{x(y)}=\alpha_{\perp, x(y)} \eta_{\perp}^{x(y)}+\eta_{\|, x(y)}, \\
f_{z}=\eta_{\|}^{z} .
\end{gathered}
$$

Since $x, y$ components of $\eta_{\|}$are undefined, we can again assume that $\eta_{\|, x}, \eta_{\|, y}, \eta_{\|, z}$ have the same correlators for the first and second moments to assure the invariance with the rotation of the system of coordinates. For $\eta_{\perp, x(y)}$ this gives the properties of Eq. (6) with an additional assumption that $\eta_{\perp, z}$ and $\eta_{\perp, x(y)}$ have the same statistical properties.

Thus both forms of the stochastic differential equations, sLLB-I and sLLB-II, are consistent with the FDT for interacting and noninteracting systems. The application of the FDT also shows its deficiency; indeed small deviations from the equilibrium imply that $\mu_{x}, \mu_{y} \ll m_{0}$; i.e., the approach is not valid for $m_{0} \rightarrow 0$.

The application of the FDT provides us with a suitable choice of uncorrelated noise variables, independent of the equilibrium $m_{0}$. However, one should note once again that in the original nonlinear LLB equation the noise is multiplicative so that the covariance matrix is dependent on current $\mathbf{m}$ : $\kappa_{i j}=\kappa_{i j}(\mathbf{m})$. To deal with a completely nonlinear problem one should turn to the FP approach.

\section{APPENDIX B: SOME USEFUL PROPERTIES OF THE STOCHASTIC FORMS OF THE LLB EQUATION}

The numerical schemes often contain the following operators $L^{j} b_{k i}$ where

$$
L^{j}=\sum_{k}^{d} b_{k j} \frac{\partial}{\partial m_{k}}
$$

which should be evaluated. ${ }^{29}$ Particularly, numerically more efficient schemes could be written for the commutative noise. The condition for the commutative noise reads

$$
L^{j} b_{k, i}=L^{i} b_{k j} \quad \forall k, i, j ;
$$

i.e., the indices $i$ and $j$ can be interchanged.

The longitudinal part of the sLLB-II equation is trivially commutative, since this part of the noise is additive. For both sLLB-I and sLLB-II equations it is easy to demonstrate that

$$
L^{j, \|} b_{l i, \|}=0, \quad L^{j, \|} b_{l i \perp} \equiv 0 .
$$

Thus the longitudinal part is always commutative. For the transverse part we have

$$
L^{j, \perp} b_{l i, \perp}=-\frac{2 D_{\perp} \alpha_{\perp}^{2} \gamma^{2}}{m^{2}}\left(\delta_{j i} m_{l}+\delta_{l j} m_{i}-\frac{2 m_{i} m_{j} m_{l}}{m^{2}}\right) .
$$

It is clear that

$$
L^{j, \perp} b_{l i, \perp} \neq L^{i, \perp} b_{l, j, \perp} .
$$

Thus it is a transverse part of the LLB which is not commutative. This is unfortunate for both LLB and LLG 
equations; the latter was noticed by Garcia-Palacios et al. ${ }^{18}$ For the sLLB-I equation additionally the mixed term is not commutative; i.e.,

$$
\begin{aligned}
L^{j, \perp} b_{l i, \|} & =\frac{2 \sqrt{D_{\|} D_{\perp}} \alpha_{\|} \alpha_{\perp} \gamma^{2}}{m^{2}}\left(\delta_{j i} m_{l}+\delta_{l j} m_{i}-\frac{2 m_{i} m_{j} m_{l}}{m^{2}}\right) \\
& \neq L^{i, \perp} b_{l, j, \|} .
\end{aligned}
$$

We interpret the stochastic LLB and LLG equations in terms of the Stratonovich calculus. The corresponding Ito equation can be derived with an additional drift term. This procedure is useful since most of numerical schemes are written in terms of the Ito calculus for which the evaluation of the stochastic integrals is straightforward. The corresponding drift terms are easy to evaluate using the known relation

$$
A_{i}^{I}=A_{i}^{S}+\frac{1}{2} \sum_{j} L^{j} b_{i j}
$$

${ }^{1}$ W. F. Brown, Phys. Rev. 135, 1677 (1963).

${ }^{2}$ W. F. Brown, IEEE Trans. Magn. 15, 1196 (1979).

${ }^{3}$ W. T. Coffey, Y. P. Kalmykov, and J. T. Waldron, The Langevin Equation with Applications in Physics, Chemistry and Electrical Engineering (World Scientific, Singapore, 1996).

${ }^{4}$ A. Lyberatos and R. W. Chantrell, J. Appl. Phys. 73, 6501 (1993).

${ }^{5}$ D. V. Berkov and N. L. Gorn, in Handbook of Advanced Magnetic Materials: Characterization and Simulation, edited by Y. Liu, J. Sellmeyer, and D. Shindo (Springer, New York, 2006), Vol. II, p. 422.

${ }^{6}$ O. Chubykalo-Fesenko, U. Nowak, R. W. Chantrell, and D. Garanin, Phys. Rev. B 74, 094436 (2006).

${ }^{7}$ G. Grinstein and R. H. Koch, Phys. Rev. Lett. 90, 207201 (2003).

${ }^{8}$ D. A. Garanin, Phys. Rev. B 55, 3050 (1997).

${ }^{9}$ N. Kazantseva, D. Hinzke, U. Nowak, R. W. Chantrell, U. Atxitia, and O. Chubykalo-Fesenko, Phys. Rev. B 77, 184428 (2008).

${ }^{10}$ U. Atxitia, O. Chubykalo-Fesenko, N. Kazantseva, D. Hinzke, U. Nowak, and R. W. Chantrell, Appl. Phys. Lett. 91, 232507 (2007).

${ }^{11}$ U. Atxitia, O. Chubykalo-Fesenko, J. Walowski, A. Mann, and M. Münzenberg, Phys. Rev. B 81, 174401 (2010).

${ }^{12}$ K. Vahaplar, A. M. Kalashnikova, A. V. Kimel, D. Hinzke, U. Nowak, R. Chantrell, A. Tsukamoto, A. Itoh, A. Kirilyuk, and T. Rasing, Phys. Rev. Lett. 103, 117201 (2009).

${ }^{13}$ N. Kazantseva, D. Hinzke, R. W. Chantrell, and U. Nowak, Europhys. Lett. 86, 27006 (2009).

${ }^{14}$ C. Schieback, D. Hinzke, M. Kläui, U. Nowak, and P. Nielaba, Phys. Rev. B 80, 214403 (2009).
For the sLLB-I case

$$
\mathbf{R}_{\mathbf{I}}=\mathbf{A}^{I}-\mathbf{A}^{\mathbf{S}}=-\frac{2 k_{\mathrm{B}} T|\gamma|}{M_{\mathrm{s}}^{0} V}\left(\alpha_{\perp}-\sqrt{\alpha_{\perp} \alpha_{\|}}\right) \frac{\mathbf{m}}{m^{2}},
$$

and for the sLLB-II

$$
\mathbf{R}_{\mathrm{II}}=\mathbf{A}^{I}-\mathbf{A}^{\mathbf{S}}=-\frac{2 k_{\mathrm{B}} T|\gamma|}{M_{\mathrm{S}}^{0} V}\left(\alpha_{\perp}-\alpha_{||}\right) \frac{\mathbf{m}}{m^{2}} .
$$

Thus the drift term is parallel to the magnetization direction. For the numerical schemes for the stochastic LLG equation this is fortunate, since the additional drift can be removed by normalizing the magnetization magnitude at each time step. Obviously, this cannot be done for the stochastic LLB equation. Note that in both cases the drift term increases with temperature and just below $T_{\mathrm{c}}$ when $m \rightarrow 0$ becomes very large. However, it becomes zero at $T>T_{\mathrm{c}}$.
${ }^{15}$ D. Hinzke and U. Nowak, Phys. Rev. Lett. 107, 027205 (2011).

${ }^{16}$ I. L. Prejbeanu, M. Kerekes, R. C. Sousa, O. Redon, B. Dieny, and J. P. Nozeires, J. Phys. Condens. Matter 19, 165218 (2007).

${ }^{17}$ F. Hendrik et al., Appl. Phys. Lett. 84, 810 (2004).

${ }^{18}$ J. L. Garcia-Palacios and F. J. Lazaro, Phys. Rev. B 58, 14937 (1998).

${ }^{19}$ T. Kamppetter, F. G. Mertenz, E. Moro, A. Sanchez, and A. R. Bishop, Phys. Rev. B 59, 11349 (1999).

${ }^{20}$ Yu. Gaididei, T. Kamppeter, F-G. Mertens, and A. Bishop, Phys. Rev. B 59, 7010 (1999).

${ }^{21}$ D. A. Garanin and O. Chubykalo-Fesenko, Phys. Rev. B 70, 212409 (2004).

${ }^{22}$ A. Rebei and M. Simionato, Phys. Rev. B 71, 174415 (2005).

${ }^{23}$ U. Atxitia, O. Chubykalo-Fesenko, R. W. Chantrell, U. Nowak, and A. Rebei, Phys. Rev. Lett. 102, 057203 (2009).

${ }^{24}$ A. Lyberatos, D. V. Berkov, and R. W. Chantrell, J. Phys. Condens. Matter 5, 193 (1993).

${ }^{25}$ O. Chubykalo, R. Smirnov-Rueda, J. M. Gonzalez, M. A. Wongsam, R. W. Chantrell, and U. Nowak, J. Magn. Magn. Mater. 266, 28 (2003).

${ }^{26}$ D. A. Garanin, Physica A 172, 470 (1991).

${ }^{27}$ D. Allen and D. J. Tyldesless, Computer Simulations of Liquids (Clarendon, Oxford, 1997).

${ }^{28}$ C. W. Gardiner, Handbook of Stochastic Methods for Physics, Chemistry and the Natural Sciences (Springer, Berlin, 2004).

${ }^{29}$ P. E. Kloeden and E. Platen, Numerical Solution of Stochastic Differential Equations (Springer, Berlin, 1995). 